YEARBOOK OF PRIVATE INTERNATIONAL LAW 



\title{
$\mathbf{S}|\mathbf{e}| \mathbf{I} \mid \mathbf{p}$ sellier european law publishers
}

\section{YEARBOOK OF PRIVATE INTERNATIONAL LAW VOLUME XIX - 2017/2018}

\author{
Founding EdITORS \\ PETAR ŠARČEVIĆ † \\ PAUL VOLKEN \\ EDITORS \\ ANDREA BONOMI \\ Professor at the \\ University of Lausanne \\ GIAN PAOLO ROMANO \\ Professor at the \\ University of Geneva
}

Associate EdITOR

ILARIA PRETELLI

Legal Adviser at the

Swiss Institute of Comparative Law

Published in Association with

SWISS INSTITUTE OF COMPARATIVE LAW

LAUSANNE, SWITZERLAND

\section{ottoschmidt}


The Deutsche Nationalbibliothek lists this publication in the Deutsche Nationalbibliografie; detailed bibliographic data are available on the Internet at http://dnb.dnb.de.

\author{
Verlag Dr. Otto Schmidt KG \\ Gustav-Heinemann-Ufer 58, 50968 Köln \\ Tel. +49 221/937 38-01, Fax +49 221/93738-943 \\ info@otto-schmidt.de,www.otto-schmidt.de \\ ISBN (print) 978-3-504-08013-6 \\ ISBN (eBook) 978-3-504-38607-8 \\ (C) 2018 by Verlag Dr. Otto Schmidt KG, Köln and \\ Swiss Institute of Comparative Law, Lausanne
}

All rights reserved. No part of this publication may be reproduced, translated, stored in a retrieval system or transmitted in any form or by any means, electronic, mechanical, photocopying, recording or otherwise, without prior permission of the publisher.

The paper used is made from chlorine-free bleached materials, wood and acid free, age resistant and environmentally friendly.

Printing and binding: Kösel, Krugzell

Printed in Germany. 


\begin{tabular}{cc}
\multicolumn{2}{c}{ ADVISORY BOARD } \\
JÜRGEN BASEDOW & HUANG JIN \\
Hamburg & Wuhan \\
Paris & THOMAS KADNER GRAZIANO \\
GENEVIÈVE BASTID-BURDEAU & Geneva \\
MICHAEL BOGDAN & EVA LEIN \\
Lund & Lausanne/London \\
SIR LAWRENCE COLLINS & HANS VAN LOON \\
London & The Hague \\
RUI MANUEL GENS DE MOURA RAMOS & YASUHIRO OKUDA \\
Lisbon/Coimbra & Tokyo \\
DIEGO P. FERNÁNDEZ ARROYO & LINDA J. SILBERMAN \\
Paris & New York \\
RICHARD FRIMPONG OPPONG & SYMEON C. SYMEONIDES \\
Kamloops (British Columbia) & Salem (Oregon) \\
LUKAS HECKENDORN URSCHELER & \\
Lausanne &
\end{tabular}

\section{Assistant Editors}

ALEXANDRA BECHEIKH

Research Assistant, University of Lausanne

ALRICK BEZAT

Research Assistant, University of Lausanne

VITO BUMBACA

Research Assistant, University of Geneva
CORINNE MAMMINO

Research Assistant, University of Lausanne

RACHEL NGO NTOMP

Research Assistant, University of Geneva

ALEXANDRE TONDINA

Research Assistant, University of Lausanne

\section{Production Assistant}

FRANÇOISE HINNI

Swiss Institute of Comparative Law

\section{ENGLISH REVISION}

CHRISTOPHER BOOTH

Research Assistant, University of Geneva
SHAHEEZA LALANI

University of Paris

VICTORIA GARRINGTON

Attorney-at-Law, Fribourg 
